\title{
AUTOMEDICAÇÃO NO TRATAMENTO DE SINTOMAS DE MANIFESTAÇÃO DE ESTRESSE POR DISCENTES DE UMA INSTITUIÇÃO FEDERAL DE ENSINO SUPERIOR NO ESTADO DO CEARÁ
}

\author{
SELF-MEDICATION IN THE TREATMENT OF STRESS MANIFESTATION SYMPTOMS BY \\ STUDENTS OF A FEDERAL HIGHER EDUCATION INSTITUTION IN THE STATE OF CEARÁ
}

Francisco Clécio da Silva Dutra ${ }^{\mathrm{a}^{*}}$

Orcid: http://orcid.org/0000-0002-3451-1664

Janiel Ferreira Felício ${ }^{\text {*** }}$

Orcid: http://orcid.org/0000-0002-5601-0086

Inara da Silva de Moura ${ }^{* *}$

Orcid: http://orcid.org/0000-0003-3612-0541

\author{
Cinthia Mendonça Cavalcante $\mathrm{d}^{\mathrm{d}^{*}}$ \\ Orcid: http://orcid.org/0000-0001-8127-1125
}

Francisco Washington Araújo Barros Nepomuceno ${ }^{\mathrm{e}^{* \star}}$

Orcid: http://orcid.org/0000-0001-9932-028X

\author{
Jeferson Falcão do Amaral ${ }^{\text {(** }}$ \\ Orcid: http://orcid.org/0000-0003-0426-0347
}

cleciouece@gmail.coma, janielferreira1@gmail.comb, inaramoura123@gmail.comc, cinthia.cavalcante@ufc.brd, barros@unilab.edu.bre,barros@ unilab.edu.br ${ }^{\mathrm{f}}$

Universidade Estadual do Ceará*, Universidade da Integração Internacional da Lusofonia Afro-Brasileira ${ }^{* *}$

Data de Submissão :07/09/2019

Data de Aceite: 14/11/2019

\section{RESUMO}

Introdução: A automedicação é uma resultante de inúmeros fatores, dentre eles se destacam a dificuldade do acesso aos serviços de saúde e a necessidade de aliviar sintomas. Os universitários são uma das conjunturas sociais que mais pratica automedicação, em que há busca por meios que amenizem perturbações causadas ou intensificadas por razões emocionais. Objetivos: Os objetivos do estudo foram avaliar a frequência da automedicação praticada e descrever quais medicamentos foram os mais utilizados pela classe discente de uma Instituição de Ensino Superior no Estado do Ceará. Métodos: Participaram da pesquisa 120 discentes. Para a coleta dos dados aplicaram-se três questionários: um questionário sobre o perfil sociodemográfico, um questionário semiestruturado a respeito da prática da automedicação e para avaliar as manifestações de estresse utilizou-se o Inventário de Sintomas de Estresse de Lipp. Resultados e Discussão: Destes, 96 alunos afirmaram fazer uso de medicamentos sem prescrição médica, correspondendo a $80 \%$ da amostra avaliada; enquanto 24 afirmaram que não, correspondendo a $20 \%$ dos discentes. Os medicamentos mais apontados foram os analgésicos e os AINES. Conclusão: $\mathrm{O}$ uso de fármacos para reversão de sintomas de manifestação de estresse é elevado nos discentes da instituição onde o presente estudo foi desenvolvido.

Palavras-chave: Automedicação; discentes; tratamento; manifestação de estresse

\begin{abstract}
Introduction: Self-medication is a result of many factors, among them the difficulty of access to health services and the need to relieve symptoms. The university students are one of the social conjunctures that practice self-medication, where the search for means that soften disturbances caused or intensified for emotional reasons. Objective: The objectives of the study were to evaluate the frequency of self-medication practiced and to describe which medications were the most used by the student class of a Higher Education Institution in the State of Ceará. Methods: 120 students participated in the research. For data collection, three questionnaires were applied: a questionnaire on socio-demographic profile, a semi-structured questionnaire about the practice of self-medication, and to assess stress manifestations, the Lipp Stress Symptom Inventory was used. Results: Of these, 96 students reported using nonprescription drugs, corresponding to $80 \%$ of the evaluated sample; while 24 said no, corresponding to $20 \%$ of the students. The most mentioned drugs were analgesics and NSAIDs. Conclusion: The use of drugs for reversal of symptoms of stress manifestation is high in the students of the institution where the present study was developed.
\end{abstract}

Keywords: Self-medication; students; treatment; manifestation of stress 


\section{Introdução}

A prática da automedicação é uma resultante de inúmeros fatores, dentre os quais se destacam a dificuldade do acesso aos serviços de saúde pela população, a crença nos benefícios do tratamento de doenças e, principalmente, a necessidade de aliviar sintomas ${ }^{1}$. Essa prática se configura como um evento potencialmente nocivo à saúde, individual e coletiva, porque é sabido que nenhum medicamento é "inofensivo" ao organismo. A utilização abusiva de drogas consideradas "banais" pela população, principalmente os analgésicos, podem trazer como consequências a resistência bacteriana, hipersensibilidade do sistema imunológico, mal-estar, náuseas, sintomas de retirada e ainda aumentar a predisponência a diversos tipos de neoplasias ${ }^{2}$.

Somado a esse cenário, a prática irracional da automedicação pode mascarar alguma doença de base, fazendo com que ela passe despercebida, progredindo em constante evolução no organismo ${ }^{3,4}$. Associado a esse cenário, há os grandes investimentos em publicidade envolvendo a comercialização dos fármacos, publicidade essa sustentada por grandes empresas do ramo ${ }^{5,6}$. A propaganda, ao difundir a percepção dos medicamentos como produtos de consumo, força, entre outras coisas, a população a praticar a automedicação, agravando doenças ou sintomas, instigando a aquisição de produtos ineficazes e/ ou inadequados e por fim ampliando gastos do Sistema de Saúde. Além disso, no que diz respeito ao tratamento de intoxicações medicamentosas, essa ocorrência ocupou, no ano de 2013, o primeiro lugar no ranking de intoxicações nos centros de controle de toxicologia e farmacovigilância do Brasil $^{7}$, devendo dessa maneira, ser tratada em um contexto mais ampliado, como um problema de saúde pública ${ }^{6}$ Dessa forma, a facilidade de acesso a medicamentos em estabelecimentos, principalmente farmácias, fazem a população confiar que os mesmos são isentos de riscos e intoxicações ${ }^{8}$. Ademais, acabam por provocar o uso indiscriminado, o que nem sempre corrobora em efeitos assim desejados, além de virem a acarretar discrepâncias no uso adequado, como o uso de medicamentos inapropriados, dose e frequência incoerentes, período de consumo insuficiente ou exacerbado em relação ao recomendado, além de interações indesejáveis com outros medicamentos ${ }^{8}$.

No atual cenário mundial, vemos um alarmante aumento de doenças de cunho psicossomático, ou seja, doenças clinicamente constatáveis causadas ou intensificadas por razões emocionais ${ }^{9}$. Dentro dessas doenças específicas podemos pontuar as manifestações de estresse como umas das doenças psicossomáticas que mais causam implicações desagradáveis na população ${ }^{10}$. As manifestações de estresse situam-se nas dimensões interativas entre o homem, o meio e a adaptação, produzindo desgastes ${ }^{11}$. Assim sendo, conviver com o ritmo de vida agitado, desgastante, com falta de tempo, tanto para o lazer como para o descanso, alimentação inadequada e excessivas cobranças são fatores fundamentais para o desenvolvimento de manifestações estressoras ${ }^{10}$.

Dentro do cotidiano dos acadêmicos, todas essas condições mencionadas são vividas, desde o ingresso à universidade. Particularidades como o regimento de horário integral do curso, o ritmo de vida intenso e a pressão psicológica determinada pelas exigências impostas pela conjuntura da instituição (professores, disciplinas, notas satisfatórias, manutenção de bolsas) e pela ansiedade pessoal de ter um bom rendimento a cada período cursado vão, aos poucos, se tornando elementos geradores de desgaste emocional nesse público ${ }^{10}$. Dentro da vivência acadêmica, os discentes passam por situações de mudança, desenvolvimento, frustrações, crescimento pessoal e intelectual, temores e angústias ${ }^{12}$. Dessa forma, constantemente se desenvolvem sentimentos como desapontamento, irritabilidade, preocupação, impaciência durante o percorrer do curso, acarretando déficit de aprendizado ${ }^{12}$.

Em decorrência dessas manifestações, outras doenças têm caminho aberto para se manifestarem; doenças essas que têm por base manifestações de estresse. Em um estudo de Souza e colaboradores ${ }^{13}$, 2015 , foi pontuado que alunos em condições de estresse buscavam alívio de dores e outros sintomas através da prática da automedicação. Os autores pontuaram que a relação entre a automedicação e as manifestações de estresse causadas pelo curso foi, em determinadas situações, confirmada pelos alunos que participaram do estudo ${ }^{13}$. 
Essa confiança em adotar a prática de se automedicar está associada à confiabilidade no que se diz respeito à formação e ao grau de escolaridade, os indivíduos com maior grau de instrução são os que mais recorrem à

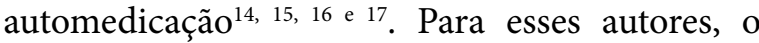
acúmulo de conhecimento, seja ele adquirido nas instituições educacionais ou em experiências de vida, gera uma maior confiança naqueles que se automedicam. Vilarino e colaboradores (1998)2, ainda acrescentam que a justificativa para a prática da automedicação está relacionada ao conhecimento adquirido, em que o mesmo pode vir a dar maior segurança a essa prática. O ideal, portanto, é utilizar o medicamento apenas quando imprescindível e recomendado por um profissional especializado 16. Dessa forma, a realização deste estudo justifica-se pela necessidade de se verificar a prática da automedicação entre estudantes de uma Instituição de Ensino Superior (IFES) no interior do Estado do Ceará. O estudo teve como objetivos avaliar a frequência da automedicação praticada e descrever quais medicamentos foram os mais utilizados pelos acadêmicos.

\section{Metodologia}

Trata-se de um estudo descritivo com abordagem quantitativa e de cunho transversal. Participaram do trabalho 120 acadêmicos devidamente matriculados em cursos de graduação em regime presencial em uma Universidade localizada no interior do Estado do Ceará. Para a coleta dos dados aplicaram-se três questionários: um questionário a respeito do perfil sociodemográfico, um questionário semiestruturado, com perguntas abertas e fechadas, a respeito da prática da automedicação, a frequência em que era realizada e ainda quais medicamentos eram utilizados e, por fim, para avaliar as manifestações de estresse utilizou-se um questionário intitulado Inventário de Sintomas de Estresse de Lipp (ISS) validado para sujeitos a partir de 15 anos ${ }^{18}$.

O ISS se constitui em um instrumento de verificação e correlação de sintomas físicos e psicológicos, divididos em 3 partes. Na primeira parte, o respondente indicou quais sintomas experimentou nas últimas 24 horas. Na segunda parte, foram assinalados os sintomas que sentiu no último mês, e, na terceira parte, os sintomas físicos e psicológicos experimentados nos últimos 3 meses. O ISS permite diagnosticar se a pessoa tem estresse, em que fase ela se encontra, e se sua sintomatologia é mais típica da área somática ou cognitiva ${ }^{19}$.

Os questionários foram aplicados após a apreciação do aluno do Termo de Consentimento Livre e Esclarecido (TCLE) e sua respectiva aceitação em participar do estudo mediante assinatura do termo. A aplicação dos questionários ocorreu entre os meses de fevereiro a maio de 2018 e foram aplicados na própria instituição, onde os estudantes encontravam-se matriculados, tal fato garantiu a total privacidade e sigilo ao sujeito da pesquisa, conforme disposto na Resolução 196/96 do Conselho Nacional de Saúde.

O processamento dos dados foi realizado por meio do programa Microsoft Excel Starter ${ }^{\circledR}$ versão 2010, na qual foi criado o banco de dados, a realização do processamento das variáveis e a criação de tabelas. $\mathrm{O}$ projeto foi submetido à apreciação pelo Comitê de Ética e Pesquisa (CEP) da Universidade da Integração Internacional da Lusofonia Afro-Brasileira - UNILAB e aprovado sob o CAAE de no 49248215.9.0000.5576.

\section{Resultados e Discussão}

Dos 120 alunos que participaram do estudo, 96 afirmaram fazer uso de medicamentos sem prescrição médica (automedicação), correspondendo a $80 \%$ da amostra avaliada, enquanto 24 afirmaram que não, correspondendo a $20 \%$, assim como indicado na Tabela 1 .

Tabela 1 - Distribuição da prática de automedicação entre os discentes.

\begin{tabular}{lcc}
\hline & $\begin{array}{c}\text { Frequência } \\
\boldsymbol{F}\end{array}$ & $\begin{array}{c}\text { Porcentagem } \\
\%\end{array}$ \\
\hline SE AUTOMEDICAM & 96 & $80 \%$ \\
$\begin{array}{l}\text { NÃO SE } \\
\text { AUTOMEDICAM }\end{array}$ & 24 & $20 \%$ \\
TOTAL & 120 & $100 \%$ \\
\hline
\end{tabular}

Fonte: Dados da pesquisa 
Um estudo semelhante realizado por Chehuen Neto e colaboradore ${ }^{17}, 2006$, em uma Universidade localizada em Juiz de Fora obteve resultados semelhantes, cerca de 75,05\% do público discente afirmou se automedicar. Esse dado corrobora para uma preocupação alarmante, pois esse público pode estar diante da eminência de Problemas Relacionados a Medicamentos (PRMs). Os PRMs podem ser ocasionados pelo uso indevido de medicamentos no combate ao estresse, o que acaba gerando impacto desta prática na saúde e no processo de ensino-aprendizagem do aluno. Os PRMs incluem diversos fenômenos: intoxicações e interações medicamentosas, reações alérgicas, superdosagens, erros na autoadministração, dentre outros $\operatorname{casos}^{20}$.

Dentro do perfil sociodemográfico, na variável gênero, pudemos identificar uma tênue diferença entre os gêneros ao praticarem automedicação. Dos 66 do sexo masculino, 52 afirmaram se automedicar, dos 54 discentes do sexo feminino, 44 afirmaram adotar essa prática. A Tabela 2 nos indica esse cenário.

Tabela 2 - Distribuição dos discentes quanto ao gênero na prática da automedicação.

\begin{tabular}{lcccc}
\hline \multirow{2}{*}{$\begin{array}{l}\text { AUTOMEDI- } \\
\text { CAÇÃO }\end{array}$} & NÃO & SIM & \multicolumn{2}{c}{ TOTAL } \\
Masculino & 14 & 52 & 66 & $\mathbf{f}$ \\
\cline { 3 - 5 } Feminino & 10 & 44 & 54 & $45 \%$ \\
TOTAL & $\mathbf{2 4 ( 2 0 \% )}$ & $\mathbf{9 6 ( 8 0 \% )}$ & $\mathbf{1 2 0}$ & $\mathbf{1 0 0 \%}$ \\
\hline
\end{tabular}

Fonte: Dados da pesquisa

Acerca do estado civil dos discentes, 81,7\% afirmaram ser solteiros, $17,5 \%$ possuem algum tipo de união estável e 1 aluno pontuou ser divorciado (a). Estudo semelhante demonstrado por Pereira, Miranda e Passos ${ }^{10}, 2010$, em uma Universidade do interior do Estado de Minas Gerais, identificou um número semelhante a respeito do percentual de discentes solteiros e casados, em que o percentual de alunos solteiros foi em demasia maior dos que possuem uma união estável (casados), onde 97\% se declararam solteiros.
Tabela 3 - Distribuição dos discentes quanto ao estado civil na prática da automedicação.

\begin{tabular}{lcccc}
\hline \multirow{2}{*}{$\begin{array}{c}\text { AUTOMEDI- } \\
\text { CAÇÃO }\end{array}$} & NÃO & SIM & \multicolumn{2}{c}{ TOTAL } \\
Solteiro (a) & 17 & 81 & 98 & $81,7 \%$ \\
Casado (a) & 6 & 15 & 21 & $17,5 \%$ \\
Viúvo (a) & 0 & 0 & 0 & $0 \%$ \\
Divorciado (a) & 1 & 0 & 1 & $0,8 \%$ \\
TOTAL & $24(20 \%)$ & $96(80 \%)$ & 120 & $100 \%$ \\
\hline
\end{tabular}

Fonte: Dados da pesquisa

A instituição onde o estudo foi realizado, configura-se, dentro de uma política que tem por embasamento a união e a cooperação entre países que possuem a Língua Portuguesa como língua oficial, intitulados como CPLP (Comunidade dos Países de Língua Portuguesa). Dessa maneira, uma das premissas dessa instituição é trazer ao país alunos advindos dos países que juntos formam esse bloco para terem sua formação universitária aqui no país. Assim sendo, um dos pontos abordados no inquérito foi avaliar se a conduta de se automedicar é diferente ou não entre os alunos de nacionalidade brasileira em comparação aos alunos estrangeiros.

$\mathrm{Na}$ amostra investigada, dos 120 alunos, 56 relataram ser estrangeiros (46,7\%) e 64 brasileiros (53,3\%). Dos 56 alunos estrangeiros, 37 afirmaram se automedicar e 19 afirmaram que não. Entre os 64 alunos brasileiros, 59 pontuaram se automedicar e apenas 5 deles negaram a adoção dessa prática. Ao analisarmos a diferença entre o número de alunos estrangeiros, em contrapartida, ao número de alunos brasileiros que se automedicam, podemos afirmar que essa prática é maior nos discentes de origem brasileira, assim como esclarecido na Tabela 4.

Tabela 4 - Distribuição dos discentes quanto à Nacionalidade na prática da automedicação.

\begin{tabular}{lcccc}
\hline AUTOMEDI- & \multirow{2}{*}{ CAÇÃO } & NÃO & SIM & \multicolumn{2}{c}{ TOTAL } \\
Brasileiros & 5 & 59 & 64 & $53,3 \%$ \\
Estrangeiros & 19 & 37 & 56 & $46,7 \%$ \\
TOTAL & $24(20 \%)$ & $96(80 \%)$ & 120 & $100 \%$ \\
\hline
\end{tabular}

Fonte: Dados da pesquisa 
Pode-seaferir dessa maneira que se automedicar se tornou um hábito rotineiro do cotidiano desses estudantes. Um estudo feito por Souza e colaboradores $^{21}, 2011$, também demonstrou uma média semelhante ao presente estudo.

$\mathrm{Na}$ avaliação das manifestações dos sintomas de estresse, realizado através do Inventário dos Sintomas de Estresse de LIPP, obtivemos um número de 86 alunos que se enquadraram em alguma das fases do inventário. Ressaltamos que, dentro do Questionário de Sintomas de Estresse de LIPP, o indivíduo pode apresentar nenhuma, uma ou mais das categorias/fases apontadas no teste, como encontramos na tabela acima. Dos 120 alunos participantes do estudo, 86 discentes $(71,7 \%)$ apresentaram manifestações de estresse em uma ou mais categorias (I, II e III) e 34 não apresentaram (28,3\%).

A Fase 1 sinaliza um ambiente de alerta e de alarme, em que o indivíduo está em contato com a fonte geradora do estresse, há relato de sensações desagradáveis, sinais e sintomas considerados leves, mas que já deixam o indivíduo em sofrimento, principalmente pelos sintomas enquadrados na psicossomática. Dentro desta fase, 5 alunos foram pontuados (4\% da amostra do estudo).

A Fase 2 engloba comportamentos caracterizados pela resistência diante dos fatores estressantes. O indivíduo assume um comportamento de luta, de fuga diante de sintomas, pois estes já lhe causam desgaste físico/emocional, e ainda algum tipo de comprometimento no seu estilo de vida, no seu cotidiano. Encontramos 80 alunos dentro desta fase $(66,6 \%)$. Podemos constatar diante desse dado que os discentes estão diante de fatores geradores de estresse e que os mesmos já estão sofrendo com sintomas da manifestação do estresse, o próprio organismo busca maneiras de se livrar desses sintomas desgastantes, tenta resistir aos malefícios provocados por essas manifestações desses sintomas, buscando retornar ao estado de equilíbrio. Nesta fase podem ocorrer: adaptação ou eliminação dos agentes estressantes, podendo ocorrer o reequilíbrio. Quando não ocorrem adaptações à manifestação dos fatores estressantes, há progressão para o próximo nível.
A Fase 3 corresponde ao aparecimento de patologias de característica crônica. Nessa ocasião o indivíduo apresenta algum nível de exaustão e de esgotamento físico/mental, é uma fase crítica ao organismo, onde há o comprometimento físico em formas de doenças. Dentro dessa fase foram encontrados 9 discentes (7,5\%). Mesmo considerando um número pequeno, devemos considerar essa amostra preocupante, pois nessa fase, como já foi mencionado, os indivíduos já demonstram doenças mais persistentes, crônicas, que causam limitações no cotidiano dos mesmos. A Tabela 5 nos demonstram esses valores.

Torna-se importante ressaltar que 8 (oito) discentes se enquadraram em mais de 2 categorias dentro do estudo.

Tabela 5 - Perfil das manifestações de sintomas de estresse de Lipp.

\begin{tabular}{lcc}
\hline CATEGORIA/FASE & $\boldsymbol{f}$ & $\%$ \\
\hline 1 & 5 & $4 \%$ \\
2 & 80 & $66,6 \%$ \\
3 & 9 & $7,5 \%$ \\
Não apresentou & 34 & $28,3 \%$ \\
TOTAL & 120 & $100 \%$ \\
\hline
\end{tabular}

Fonte: Dados da pesquisa

Portanto, almejamos com essas informações, correlacionar as possíveis manifestações de sintomas de estresse com a prática da automedicação, pois muitos sinais clínicos do estresse advêm de sinais e sintomas considerados “insignificantes" por grande parte da população. Assim sendo, sintomas clássicos de manifestações advindas do estresse como cefaleias, mal-estar, enjoos, quadros de vômitos, insônia, pesadelos, sono ineficaz, desconfortos estomacais/gastrite, dores musculares, constipação, episódios diarreicos, pensamentos negativos em torno de si mesmo, autonegação, pensamentos de inferioridade e de incompetência são os sinais que mais levaram os alunos a praticarem a automedicação. O problema em torno de fazer uso de medicamentos para buscar resolver esses sintomas pode, na grande maioria das vezes, mascarar determinadas doenças, que podem se desenvolver e se alastrarem pelo organismo silenciosamente ${ }^{12}$. 
Estudo realizado por Damasceno, Terra, Zannetti et al. ${ }^{22}, 2007$, constatou que os principais motivos que levaram os acadêmicos estudados à automedicação foram: dor de cabeça $(35,6 \%)$; dores de modo geral (13,4\%); febre $(12,4 \%)$ e dor de garganta $(5,8 \%)$. Agrupando-se os casos relacionados à dor (dor de cabeça, dores e dor de garganta), esta apareceu como o principal motivo que levou os graduandos a praticarem a automedicação, (54,8\%).

Um outro estudo, realizado por Shankar, Partha e Shenoy ${ }^{23}$, em 2002, pontuou a dor de cabeça e a febre, que juntas contabilizaram $60,0 \%$ dos principais sintomas/doenças que levaram à automedicação. Em um outro inquérito apresentado por $\operatorname{Vitor}^{24}, 2008$, pode-se notar que houve um predomínio de entrevistados $(66,03 \%)$ nos casos em que a ocasião mais comum de automedicação também foi a dor de cabeça. A dor de cabeça configura-se em inúmeros quadros clínicos de sinais e sintomas de múltiplas patologias, mascará-la com a prática de se automedicar merece precaução e atenção. Diante desse cenário, é notória a preocupação com os dados que o presente estudo nos demonstra como também dos estudos citados anteriormente acerca da finalidade que a prática da automedicação está sendo norteada.

Para identificação das classes terapêuticas de medicamentos utilizados pelos acadêmicos para aliviar sintomas de estresse, os fármacos foram classificados através do nível $2 / 3$ do uso do sistema de Anatomical Therapeutic Chemical (ATC) da Organização Mundial da Saúde (OMS). No sistema ATC (classificação anatômica, terapêutica e química), os medicamentos são dispostos em diferentes grupos de acordo com seus sítios de ação, e suas características terapêuticas e químicas. Existem cinco níveis diferentes: os medicamentos são divididos em 14 grupos anatômicos principais (nível 1), os quais abrigam dois subgrupos terapêutico/farmacológicos (níveis 2/3), o nível 4, subgrupo terapêutico/farmacológico/ químico; e o nível 5, a substância química propriamente $\operatorname{dita}^{25}$.

Tabela 6 - Perfil medicamentoso (automedicação) conforme níveis 2/3 da classificação ATC

\begin{tabular}{|c|c|c|c|}
\hline FÁRMACOS & $\begin{array}{l}\text { Masculino } \\
f\end{array}$ & $\begin{array}{l}\text { Feminino } \\
F\end{array}$ & $\begin{array}{l}\text { Total } \\
f(\%)\end{array}$ \\
\hline \multicolumn{4}{|l|}{ Antiácidos - A02A } \\
\hline Omeprazol & 4 & 3 & $7(4 \%)$ \\
\hline $\begin{array}{l}\text { Hidróxido de alumínio + } \\
\text { Hidróxido de magnésio + simeticona }\end{array}$ & 0 & 1 & $1(0,5 \%)$ \\
\hline \multicolumn{4}{|l|}{ Antiespasmódicos - a03 } \\
\hline Escopolamina + dipirona & 5 & 2 & $7(4 \%)$ \\
\hline \multicolumn{4}{|l|}{ Antidiarreicos - A07 } \\
\hline Saccharomyces boulardii-17 liofilizado & 1 & 0 & $1(0,5 \%)$ \\
\hline \multicolumn{4}{|l|}{ Antitrombóticos- B01 } \\
\hline Ácido acetilsalicílico & 1 & 2 & $3(2 \%)$ \\
\hline \multicolumn{4}{|l|}{ Agentes Beta-Bloqueadores - C07 } \\
\hline Propanolol & 1 & 0 & $1(0,5 \%)$ \\
\hline \multicolumn{4}{|l|}{ Antagonista de angiotensina II - C09CA } \\
\hline Losartana & 0 & 2 & $2(1 \%)$ \\
\hline \multicolumn{4}{|l|}{ Urológicos - G04 } \\
\hline Finasterida & 1 & 0 & $1(0,5 \%)$ \\
\hline \multicolumn{4}{|l|}{ Antimicrobianos - J01 } \\
\hline Cefalexina & 1 & 1 & $2(1 \%)$ \\
\hline
\end{tabular}




\begin{tabular}{llll}
\hline FÁRMACOS & Masculino & Feminino & $\begin{array}{l}\text { Total } \\
f(\%)\end{array}$ \\
\hline Sulfametoxazol & $f$ & $\boldsymbol{F}$ & $2(1 \%)$ \\
AINES - M01A & 1 & 1 & $7(4 \%)$ \\
Ibuprofeno & & 5 & $2(1 \%)$ \\
Nimesulida & 2 & 2 & $2(1 \%)$ \\
Ácido mefenâmico & 0 & 2 & $61(33,3 \%)$ \\
Analgésicos e antipiréticos - N02B & 0 & $68(37 \%)$ \\
Dipirona & & 23 & $7(4 \%)$ \\
Paracetamol & 38 & 22 & $1(0,5 \%)$ \\
Dipirona + orfenadrina + cafeína & 46 & 3 & $6(3,2 \%)$ \\
Anti-helmínticos - P02 & 4 & & \\
Albendazol & & 0 & $2(1 \%)$ \\
Antibióticos - G01AA & 1 & 2 & $183(100 \%)$ \\
Amoxicilina & & 2 & \\
Antiasmáticos - R03 & 4 & 73 & \\
Prometazina & & 0 & \\
TOTAL & 110 & & \\
\hline
\end{tabular}

Fonte: Dados da pesquisa

Dos 19 medicamentos usados com mais frequência pelos acadêmicos, a classe dos analgésicos e antipiréticos foi mais prevalente com $74,3 \%$, sendo o paracetamol o medicamento mais utilizado desta classe com $37 \%$, seguido pela dipirona com 33,3\%. Os estudos $1,22,12,16,15$, 26 e 3 encontraram resultados semelhantes, nos quais os medicamentos mais utilizados foram, em unanimidade, a classe dos analgésicos, mais especificamente os medicamentos Paracetamol e a Dipirona.

Esse alto consumo dessas drogas justificase pelo fato de serem de livre acesso nos estabelecimentos farmacêuticos aliado ao pensamento errôneo da população de que são isentos de efeitos nocivos ao organismo, essa junção tem aumentado significativamente $o$ número de intoxicações por esses fármacos, principalmente o Paracetamol ${ }^{27,28}$. Vale destacar também o efeito hepatotóxico que o Paracetamol apresenta, a toxicidade do Paracetamol, em torno das células do tecido hepático (hepatócitos), pode ocorrer por três maneiras, vindo a acontecer de maneira independente ou em associação. A forma mais comum de hepatotoxicidade é a "overdose" (ingestão de doses superiores a $10 \mathrm{~g}$ em adultos e até $150 \mathrm{mg} / \mathrm{kg}$ em crianças). O outro mecanismo é a situação de excessiva ativação do sistema citocromo P450 (CYP), resultante do uso de determinados medicamentos indutores enzimáticos e álcool crônico, e o terceiro é através da depleção dos níveis de glutation do hepatócito por ingestão alcoólica e desnutrição ${ }^{29}$. Assim sendo, podemos atribuir as maiores causas de episódios de insuficiência hepática às intoxicações por Paracetamol ${ }^{28}$.

A segunda classe dos fármacos mais prevalentes foi dos anti-inflamatórios não esteroidais (AINES), com $6 \%$, sendo o Ibuprofeno o mais utilizado, com 4\%. $\mathrm{Na}$ literatura dados semelhantes são encontrados em diversos trabalhos ${ }^{30,31,29,}$ encontraram um cenário semelhante em que os AINES (anti-inflamatórios) ocuparam a segunda posição dentre os fármacos mais utilizados na prática da automedicação. Também foi observado o uso de antiácidos ocupando a terceira posição dos fármacos mais prevalentes na amostra, com 4,5\%, sendo Omeprazol 4\%.

Podemos pontuar que não se pode condenar o hábito de se automedicar, porque esse fato seria economicamente inviável, tendo em vista não haver a real necessidade de atendimento de 
um médico para a solução de todos e quaisquer os sintomas da população ${ }^{2}$. É, por diversas justificativas, impossível frear essa prática. Dessa forma, é necessário que a sociedade se adapte, recebendo informação científica de qualidade sobre os medicamentos de venda-livre, para que se tornem imponderadas, sem estímulo ao consumo desenfreado ou ao mito de uma cura milagrosa e rápida, ao mesmo tempo que seja incentivada sempre à procura do profissional médico.

\section{Conclusões}

O uso de fármacos para reversão de sintomas de manifestação de estresse é consideravelmente elevado nos discentes da instituição onde o presente estudo foi desenvolvido. Existe, dessa forma, uma preocupação com o atual quadro da automedicação praticada por esses acadêmicos.

Foi possível evidenciar também o elevado uso de analgésicos, caso do Paracetamol e AINES, como o Ibuprofeno. Destaca-se como um quadro alarmante, pois os índices de possíveis intoxicações se tornam maiores e mais fáceis de virem a ocorrer.

A partir destas afirmativas, faz-se necessário que novas pesquisas farmacoepidemiológicas sejam aplicadas com o intuito de aferir e investigar a automedicação praticada por esses alunos e, dessa forma, produzir intervenções no sentido de alertar o público universitário acerca dos riscos associados à automedicação, com vistas a evitar que o uso indiscriminado de medicamentos se amplie nas Universidades.

Por fim, o uso abusivo de medicamentos ainda configura uma realidade que precisa ser modificada no cotidiano das Universidades, na perspectiva não só de atender às regulamentações propostas, mas de interferir positivamente na vida e na saúde dos estudantes e consequentemente na experiência profissional pós Universidade. Espera-se que os resultados deste trabalho contribuam para a ampliação dos conhecimentos sobre os impactos socioculturais e de saúde que as manifestações de estresse e, consequentemente, a automedicação, podem causar no desempenho intelectual do corpo discente da instituição. Assim como fornecer dados à gestão superior, para que possam utilizá-los como base para medidas de melhoria do bem-estar de seus universitários.

\section{Referências}

1. Arrais PS, Coelho HL, Batista MC, Carvalho ML, Righi RE, Arnau JM. Perfil da automedicação no Brasil. Rev. Saúde Pública. 1997; 31(1):71-7.

2. Vilarino JF, et al. Perfil da automedicação em município do Sul do Brasil. Rev. Saúde Pública. 1998; 32(1): 43-9.

3. Lopes WDFL, Coelho MRDOM, Oliveira JP, Oliveira Araujo YM, Melo MDCN, \& Tapety FI. A prática da automedicação entre estudantes de uma instituição de ensino superior de Teresina-PI. Revista Interdisciplinar. 2014; 7(1):17-24.

4. Minatti-Hannuch SN, Smith RL, Guimarães AS, MestreRosa VL, Marques SES. Uso de substâncias para alívio imediato da dor (SAID) em pacientes com cefaleia: estudo em uma população ambulatorial. Rev Assoc Med Bras. 1992; 38:17-23.

5. Rabello ET, \& Camargo Júnior KRD. Propagandas de medicamentos: a saúde como produto de consumo. Interface-Comunicação, Saúde, Educação. 2012; (16):557-67.

6. Batista AM, Carvalho MCRD. Avaliação da propaganda de medicamentos veiculada em emissoras de rádio. Ciência \& Saúde Coletiva. 2013; 18(2):553-61.

7. SINITOX - Sistema Nacional de Informações TóxicoFarmacológicas. Fundação Oswaldo Cruz - Ministério da Saúde - 2009. [acesso em 2019 mar 22]. Disponível em: https://sinitox.icict.fiocruz.br/.

8. Barros ARR, Griep RH, Rotenberg L Self-medication Among Nursing workers from Public Hospitals. Rev Latino-am Enfermagem. 2009; 17(6):1015-22.

9. Martani S. Doença Psicossomática: desvende esse mistério e saia do estresse. (2006). [acesso em 2016 nov 22]. Disponível em: http://www.revistahospitaisbrasil. com.br.

10. Pereira CDA, Miranda LCDS, \& Passos JP. O estresse e seus fatores determinantes na concepção dos graduados de enfermagem. Revista Mineira de Enfermagem. 2010; 14(2): 204-9.

11. Limongi-França AC. (2008). Psicologia do trabalho: psicossomática, valores e práticas organizacionais. Saraiva.

12. Monteiro CFS, Freitas JFM, \& Ribeiro AAP. Estresse no cotidiano acadêmico: o olhar dos alunos de enfermagem da Universidade Federal do Piauí. Escola Anna Nery Revista de Enfermagem. 2007; 11(1), 66-72. 
13. Souza MA, Hoeller B, Goetz ER. Estudo comparativo da automedicação praticada por estudantes dos cursos das áreas de Ciências da Saúde, Humanas, Exatas e Sociais da Universidade do Planalto Catarinense - UNIPLAC. Rev. Infarma, Ciências Farmacêuticas. 2015; 27(2): 142-8.

14. Galato D, Madalena J, Pereira G B. Self-medication among university students: the influence of the field of study. Ciênc. Saúde coletiva [online]. 2012; 17(12): 332330. [acesso em 2019 mar 22]. Disponível em: http:// dx.doi.org/10.1590/S1413-81232012001200017.

15. Silva LSF, et al. Automedicação em acadêmicos de cursos de graduação da área da saúde de uma universidade privada do Sul do Estado de Minas Gerais. Odontol. Clín.-Cient. 2011; 10(1): 57-63.

16. Aquino DS, Barros JA, Silva MDP. A automedicação e os acadêmicos da área de saúde. Ciência \& Saúde Coletiva. 2010; 15(5): 2533-8.

17. Chehuen NJA. et al. Automedicação entre Estudantes da Faculdade de Medicina da Universidade Federal de Juiz de Fora. HU rev, Juiz de Fora. 2006; 32(3): 59-64.

18. Lipp MEN, Tanganelli MS. Stress e qualidade de vida em magistrados da justiça do trabalho: diferenças entre homens e mulheres. Psicologia: reflexão e crítica. 2002; 15(3): 537-48.

19. Lipp MEN. Stress e suas implicações. Estudos de Psicologia, Campinas. 1984; 1(3-4): 5-19.

20. Fernandez-Llimos F, Faus MJ. Importance of medicinerelated problems as risk factors. The Lancet. 2003; 362(9391):1239.

21. Souza LAF, et al. Prevalência e caracterização da prática de automedicação para alívio da dor entre estudantes universitários de enfermagem. Rev. Latino-Am. Enf. $2011 ; 19(2)$.

22. Damasceno DD, Terra FDS, Zanetti HHV, D’Andréa ÉD, Silva $\mathrm{H}$, Leite A. Automedicação entre graduandos de enfermagem, farmácia e odontologia da Universidade Federal de Alfenas. REME. 2007; 11(1), 48-52.

23. Shankar PR, Partha P, Shenoy N. Self-medication and non-doctor prescription practices in Pokhara valley, Western Nepal: a questionnaire-based study. BMC Family Practice 2002; 3(17):29-35.
24. Vitor RS, Lopes CP, Menezes HS, Kerkhoff CE. Padrão de consumo de medicamentos sem prescrição médica na cidade dePorto Alegre, RS. Ciênc. saúde coletiva [Internet]. 2008 Abr [acesso em 2019 Mar 23]; 13:737-43. Disponível em: $\quad$ http://www.scielo.br/scielo.php?script=sci arttext\&pi3-81232008000700024\&lng=en. http://dx.doi. org/10.1590/S1413-81232008000700024.

25. Brasil. Portaria no 1.254, de 29 de julho de 2005 . Atualização da Relação Nacional de Medicamentos Essenciais. - Rename. Diário Oficial da União 2005; 29 mar. [acessado 2019 mar 22]. Disponível em: http://bvsms.saude.gov.br/bvs/saudelegis/gm/2005/ prt1254_27_07_2005.html

26. Telles Filho PCP, Pereira Júnior AC. Automedicação em crianças de zero a cinco anos: fármacos administrados, conhecimentos, motivos e justificativas. Esc. Anna Nery [Internet]. 2013 jun. 17(2): 291-97. [acesso em 2019 mar 22]. Disponível em: http://www.scielo. br/scielo.php?pi81452013000200013\&script $=$ sci_ abstract\&tlng=pt

27. Sebben VC, et al. Validação de metodologia analítica e estudo de estabilidade para quantificação sérica do paracetamol. Rev. Bras. Patol. Med. Lab. 2010; 46(2): 143 8.

28. Lopes J, Matheus ME. Risco de hepatotoxicidade do Paracetamol (Acetaminofem). Rev. Bras. Farm. 2012; 93(4):411-14.

29. Júnior ACP, Filho PCPT, Azevedo DSS. Automedicação: consumo, orientação e conhecimento entre acadêmicos de enfermagem. Rev enferm UFPE [on line]. 2013; 7(6): 4472-8. [acesso em 2019 mar 22]. Disponível em: https:// periodicos.ufpe.br/revistas/revistaenfermagem/

30. Masson W, Furtado PL, Lazarini CA, Oliveira CL. Automedicação entre acadêmicos do curso de medicina da Faculdade de Medicina de Marília, São Paulo. Revista Brasileira de Pesquisa em Saúde/Brazilian Journal of Health Research. 2012; 14(4).

31. Silva RCS, et al. Automedicação em acadêmicos do curso de medicina. Medicina (Ribeirão Preto). 2012; 45(1): $5-11$.

\section{Como citar este artigo:}

Dutra FCS, Felício JF, Moura IS, Cavalcante CM, Nepomuceno FWAE, Amaral JF. Automedicação no tratamento de sintomas de manifestação de estresse por discentes de uma instituição federal de ensino superior no estado do Ceará. Rev. Aten. Saúde. 2019; 17(62): 81-89. 